\title{
Impact of Femoral Stem Prosthesis Tip On Femoral Cortex After Primary Total Hip Arthroplasty (THA)
}

\section{Yang Zhang}

wannan yi xue yuan di yi fu shu yi yuan: Yijishan Hospital of Wannan Medical College https://orcid.org/0000-0001-9618-9781

Qiang Wang ( 13909636163@126.com )

wannan yi xue yuan di yi fu shu yi yuan: Yijishan Hospital of Wannan Medical College

Qi Cheng

wannan yi xue yuan di yi fu shu yi yuan: Yijishan Hospital of Wannan Medical College

Dasai Wang

wannan yi xue yuan di yi fu shu yi yuan: Yijishan Hospital of Wannan Medical College

Jian Cheng

wannan yi xue yuan di yi fu shu yi yuan: Yijishan Hospital of Wannan Medical College

Chao Wang

wannan yi xue yuan di yi fu shu yi yuan: Yijishan Hospital of Wannan Medical College

\section{Congming Li}

wannan yi xue yuan di yi fu shu yi yuan: Yijishan Hospital of Wannan Medical College

Pingbo Chen

wannan yi xue yuan di yi fu shu yi yuan: Yijishan Hospital of Wannan Medical College

Yongzhi Wu

wannan yi xue yuan di yi fu shu yi yuan: Yijishan Hospital of Wannan Medical College

\section{Research article}

Keywords: Total hip arthroplasty, impingement, femoral prosthesis, post-operative thigh pain

Posted Date: September 21st, 2021

DOI: https://doi.org/10.21203/rs.3.rs-885668/v1

License: (c) (1) This work is licensed under a Creative Commons Attribution 4.0 International License.

Read Full License 


\section{Abstract}

Background: The mechanisms of pain after total hip arthroplasty (THA) is not clear, which may be related to its impact on the femoral cortex caused by improper prosthesis placement. This study was to explore the the impact of the femoral stem prosthesis on the femoral cortex after implantation, and its relationship with postoperative thigh pain.

Methods: Totally 172 patients who underwent primary THA were retrospectively analyzed, who were divided into the impact $(n=25)$ and non-impact $(n=147)$ groups. Contact or penetration of the femoral stem prosthesis tip with the distal femoral cortex was defined as impingement. Patients were followed up and recorded.

Results: In the patients undergoing primary THA, 25 patients (14.5\%) had impact, including 7 males and 18 females. Most of the patients in the impact group were women of small stature. In the impact group, the postoperative thigh pain was more obvious, and the Harris Hip score (HHS) was lower, compared with the non-impact group $(P<0.05)$. The regression analysis showed that height $(\mathrm{OR}=1.196 ; 95 \% \mathrm{Cl}$ : 1.059 $1.351 ; \mathrm{P}=0.004)$ and prosthesis implant angle $(\mathrm{PIA})(\mathrm{OR}=0.208 ; 95 \% \mathrm{Cl}: 0.074-0.584 ; \mathrm{P}=0.003)$ were important risk factors for distal femoral cortical impingement in patients who underwent THA.

Conclusions: Patients with short stature should have adequate preoperative planning for primary THA, to prevent increased risk of postoperative thigh pain due to impinging femoral stem implants.

\section{Introduction}

In recent years, the application of biotype femur stem for primary total hip arthroplasty (THA) has achieved great success in clinic, especially for young and active patients.[1-3] Our previous study has shown that improper placement of the femoral stem prosthesis would often lead to the collision between the femoral stem tip and the femoral cortex. For long time period, the femoral stem prosthesis and femoral cortical contact might lead to bone dissolving, prosthesis loosening and fracture around the prosthesis. Moreover, the incidences of complications would also be increased, especially in the Asian crowd in Asia, with commonly seen femur arch deformity. The femoral stem prosthesis and femoral matching degree is not enough, which would lead to postoperative discomfort.[4] One of the causes of the discomfort is the mid-thigh pain, and this particular anterolateral pain in the middle of the thigh [5] may be due to the overloading or fretting of the prosthesis stem tip ,[6, 7] probably involving the placement of the acetabular and femoral stem prosthesis . $[4,8-10]$ In these studies, the femur model was established by mimics 20.0(Mimics Medical ,version 20.0), and the LK-LCU(LCU-PoroLink) femoral stem prosthesis model was extracted and implanted into the femur. Then the ANSYS19.2 was imported with 343-N force vertically downward in the center of the femoral head, and the force on the femur was compared between the patients with and without impact. It has been shown that the model with hollow tip has uniform force and no large stress concentration. In the model of tip impact, there would be local stress concentration (Fig. 1). Although it might not lead to femoral deformation, the stress concentration 
further verifies the possibility of the postoperative pain. The mechanism of pain after primary THA are still needed to be elucidated.

Current studies have mainly focused on the location of the acetabular prosthesis in THA, while few studies have concerned the location of the femoral stem prosthesis.[11, 12] Based on previous findings, we hypothesized that the femoral stem tip would impact on the femoral cortex and induce stress concentration, resulting in postoperative thigh pain. In this study, the impact of the femoral stem tip on the distal femoral cortex, as well as the impact on the thigh pain, were investigated, in patients after primary THA.

\section{Materials And Methods}

\section{Study design and patients}

Totally 172 patients who underwent primary total hip arthroplasty (THA) were included in this study and

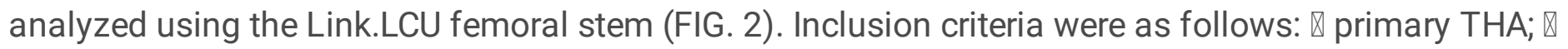
complete basic and radiography data; $\nabla$ follow-up period no less than 12 months; and $\otimes$ clear awareness and active cooperation. Exclusion criteria were as follows: $\otimes$ bilateral total hip replacement; $₫$ patients with fuzzy basic data or imaging data; and $\otimes$ patients who lost access or were unwilling to cooperate (Fig. 3 ). Young et al. [13] have believed that the structure of the femur on both sides of the normal human body was roughly symmetrical, and therefore the data herein were not subdivided. Barrack et al. [14] have suggested that postoperative thigh pain exists when patients have pain in the anterior and/or lateral thighs below the inguinal region. These 172 patients were routinely followed up at 3,6 , and 12 months post-operation. At each follow-up evaluation, patients' Harris Hip Scores (HHS) were assessed, and they were asked about the thigh pain. The intensity of thigh pain, if present, was measured using the Visual Analogue Scale (VAS).

\section{Operative process and radiographic evaluation}

\section{Operative process}

All operations were performed by the same experienced chief orthopedic surgeon using a posterolateral gluteal approach, under general or epidural anesthesia, in a healthy lateral decubitus position. The skin and subcutaneous tissue were dissected to expose the capsule, and the hip joint was adducted and rotated internally. A femoral neck osteotomy was conducted to fully expose the acetabulum and the surrounding capsule was cleared. The acetabulum was filed down to place the biological acetabular component at an appropriate combined anteriorly angle. The proximal femur was opened, the femoral bone marrow cavity was filed to an appropriate size with a medullary cavity file, and the femoral stem prosthesis was installed with a forward tilt of $15^{\circ}-20^{\circ}$. The artificial femoral head was installed and the hip joint was reset. The incision was inspected and rinsed, drainage tube was placed, and the incision was sutured layer by layer. 


\section{Radiographic evaluation}

Postoperative anteroposterior and lateral X-rays of the hip joint were observed by three orthopedic surgeons through the medical image archive and communication system. The contact or penetration of the femoral stem tip with the distal femoral cortex was defined as impingement (Figure 4a). Preoperative DR radiography of the hip was taken. According to the canal flare index (CFI) classification of Noble and Dorr et al.: A, 『4.7, champagne-fluted; B, 3.0-4.7, normal; and C, $₫ 3.0$, stovepipe (Figure 4b).[15, 16] The angle between the shaft axis of the femoral shaft and the long axis of the femoral stem was measured on the anteroposterior radiographs of the hip joint after surgery, that was the prosthesis implant angle (PIA) $\left(>3^{\circ}\right.$ or $\left.\leq 3^{\circ}\right)$ (Figure $4 \mathrm{c}$ ). According to the definition of impact, patients were divided into the following two groups: the impact $(n=25)$ and non-impact $(n=147)$ groups. Demographic and clinical data of the included patients were shown in Table 1.

\section{Statistical analysis}

The SPSS software (version 25.0) was used for the descriptive analysis of variables. Quantitative data were expressed as mean \pm SD. Classified data were expressed as absolute frequency and relative frequency. Univariate significance of comparison between collision and non-collision groups was established with the independent sample T-test of continuous variables or non-parametric test of independent samples, and the chi-square test of classified variables. With the logistic regression, the odds ratio (OR) and 95\% confidence interval (Cl) were used to represent the risk factors of cortical impingement. $P<0.05$ was considered as statistically significant.

\section{Results}

A total of 150 patients with primary THA were retrospectively analyzed in this study, including 73 males $(48.7 \%)$ and 77 females (51.3\%). The average age was $67.3 \pm 12.3$ years old, ranging from 26 to 92 years old. Patients were divided into the impact and non-impact groups, with $19(12.7 \%)$ patients in the impact group and 131 (87.3\%) patients in the non-impact group. By comparing the clinical and imaging parameters of these two groups, our results showed that there were significant differences in gender, height and placement angle between these two groups (Table 2). In the impact group, 14 cases were female (73.7\%) and 5 cases were male (26.3\%). After the primary total hip replacement, the incidence of cortical impact on the distal femur in females was significantly higher than the males $(P<0.05)$. In the impact group, $7(36.8 \%)$ patients were with implant angle $>3^{\circ}$ while $12(63.2 \%)$ patients were with implant angle $\leq 3^{\circ}$. The incidence of cortical impact in the distal femur after the primary total hip replacement was significantly higher in patients with implant angle $>3^{\circ}$ than those with implant angle $\leq 3^{\circ}(P<0.05)$. In addition, the average height of patients in the impact group was lower than the non-impact group $(P<$ 0.05). Other parameters, including the age, body mass, body mass index, length of femoral stem and configuration of femoral bone marrow cavity, had no significant difference between the impact and nonimpact groups $(P>0.05)$. Binary logistic regression analysis was further performed to explore the risk factors of distal femoral cortical impingement. The regression equation showed that the height $(\mathrm{OR}=$ 
$1.144 ; 95 \% \mathrm{Cl}, 1.010-1.295 ; P=0.034)$ and the implant angle (OR $=0.159 ; 95 \% \mathrm{Cl}, 0.044-0.575 ; P=0.005)$ were important risk factors for distal femoral cortical impingements in patients undergoing total hip replacement. Gender $(\mathrm{OR}=1.993 ; 95 \% \mathrm{Cl}, 0.462-8.594 ; P=0.355)$ was not an important risk factor for distal femoral cortical impingement in patients with primary total hip replacement (Table 3 ). In addition, during the postoperative follow-up, the postoperative pain and Harris score in the impact group were significantly lower than the non-impact group, at 3 months after surgery $(P<0.05)$. At 6 and 12 months, there were no significant differences in postoperative pain and Harris score between these two groups ( $P$ $>0.05$ ) (Table 4). The specific distribution of VAS population is shown in Fig. 5. Most patients have no pain after 3 months; A small number of patients have mild pain but it does not affect work and life; Very few patients start to have pain after walking a little more in a short period of time, but they can tolerate it, and get better after rest; Only one patient had postoperative infection and severe pain, which affected work and life. He came to the hospital for treatment. After two weeks of treatment, the pain improved.

Table 1

Data of study patients.

\begin{tabular}{|ll|}
\hline Gender $($ male $=73$ / female $=77)$ & \\
\hline Medullary cavity shape $(A=22 / B=99 / C=31)$ & \\
\hline Prosthesis implant angle $(\mathrm{PIA})$ & \\
$\left(>3^{\circ}=21 / \leq 3^{\circ}=130\right)$ & $67.31 \pm 12.32$ \\
\hline Age $($ years $)$ & $164.69 \pm 5.01$ \\
\hline Height $(\mathrm{cm})$ & $58.55 \pm 8.46$ \\
\hline Weight $(\mathrm{kg})$ & $21.60 \pm 3.06$ \\
\hline BMl $\left(\mathrm{kg} / \mathrm{m}^{2}\right)$ & $141.37 \pm 10.85$ \\
\hline Femoral stem length $(\mathrm{mm})$ & \\
\hline
\end{tabular}

Notes: A was the champagne glass type, B was the normal type, while C was the chimney type. 
Table 2

Relationship between different influencing factors and cortical impingement of distal femur.

\begin{tabular}{|llll|}
\hline Parameter & $\begin{array}{l}\text { Impact group } \\
(\mathbf{n}=19)\end{array}$ & $\begin{array}{l}\text { Non-impact group } \\
(\mathbf{n}=131)\end{array}$ & $\boldsymbol{P}$ \\
\hline Gender (female/male) & $\mathbf{1 4 / 5}$ & $\mathbf{6 3 / 6 8}$ & 0.037 \\
\hline Age (years) & $\mathbf{6 7 . 3} \pm 14.6$ & $\mathbf{6 7 . 3} \pm 12.0$ & 0.985 \\
\hline Height $(\mathrm{cm})$ & $\mathbf{1 6 0 . 8} \pm \mathbf{5 . 8}$ & $\mathbf{1 6 5 . 2} \pm \mathbf{4 . 7}$ & $\mathbf{0 . 0 0 0}$ \\
\hline Weight $(\mathrm{kg})$ & $\mathbf{5 6 . 9} \pm 10.4$ & $\mathbf{5 8 . 8} \pm \mathbf{8 . 2}$ & $\mathbf{0 . 3 6 4}$ \\
\hline BMI $\left(\mathrm{kg} / \mathrm{m}^{2}\right)$ & $\mathbf{2 2 . 1} \pm \mathbf{4 . 1}$ & $\mathbf{2 1 . 5} \pm 2.9$ & $\mathbf{0 . 6 0 9}$ \\
\hline Femoral stem length $(\mathrm{mm})$ & $\mathbf{1 3 9 . 7} \pm 14.1$ & $\mathbf{1 4 1 . 6 \pm 1 0 . 3}$ & $\mathbf{0 . 4 8 5}$ \\
\hline Medullary cavity shape $(\mathrm{A} / \mathrm{B} / \mathrm{C})$ & $3 / 9 / 7$ & $19 / 90 / 24$ & 0.246 \\
\hline Prosthesis implant angle $(\mathrm{PIA})\left(>3^{\circ} / \leq 3^{\circ}\right)$ & $7 / 12$ & $13 / 118$ & 0.001 \\
\hline
\end{tabular}

Notes: A was the champagne glass type, $B$ was the normal type, and $C$ was the chimney type.

Table 3

Influencing factors of cortical impingement of distal femur.

\begin{tabular}{|lllll|}
\hline Variables & B & SE & \multicolumn{2}{l|}{ Multivariate analysis } \\
\cline { 5 - 6 } & & & OR $(95 \%$ Cl $)$ & $P$ \\
\hline Gender & 0.690 & 0.746 & $1.993(0.462-8.594)$ & 0.355 \\
\hline Height & 0.134 & 0.063 & $1.144(1.010-1.295)$ & 0.034 \\
\hline Prosthesis implant angle (PIA) $\left(>3^{\circ} / \leq 3^{\circ}\right)$ & -1.840 & 0.656 & $0.159(0.044-0.575)$ & 0.005 \\
\hline
\end{tabular}


Table 4

Postoperative thigh pain and Harris Hip score.

\begin{tabular}{|llll|}
\hline Parameter & $\begin{array}{l}\text { Impact group } \\
(\mathbf{n}=\mathbf{1 9})\end{array}$ & $\begin{array}{l}\text { Non-impact group } \\
(\mathbf{n}=\mathbf{1 3 1})\end{array}$ & $P$ \\
\hline Postoperative thigh pain (pain/ no pain) & & & \\
\hline At 3 months after surgery & $5 / 14$ & $10 / 121$ & 0.011 \\
\hline At 6 months after surgery & $2 / 17$ & $4 / 127$ & 0.120 \\
\hline At 12 months after surgery & $1 / 18$ & $2 / 129$ & 0.277 \\
\hline Harris Hip scores & & & \\
\hline At 3 months after surgery & $79.21 \pm \mathbf{4 . 4 7}$ & $81.81 \pm 4.68$ & 0.024 \\
\hline At 6 months after surgery & $83.79 \pm \mathbf{5 . 3 7}$ & $85.69 \pm 4.90$ & 0.120 \\
\hline At 12 months after surgery & $86.05 \pm \mathbf{5 . 5 1}$ & $87.85 \pm \mathbf{4 . 7 8}$ & 0.134 \\
\hline
\end{tabular}

\section{Discussion}

As is known to all, postoperative thigh pain is a common problem after the primary total hip arthroplasty (THA) with cementless prosthesis, which would be one of the main reasons for decreased surgical satisfaction. $[10,17]$ Although the conical femoral stem prosthesis could reduce the pain occurrence, it still has a high incidence due to unknown mechanisms. $[18,19]$ At present, researches on impact after THA between prosthesis, strikes and iliopsoas muscle (between the bones and abundant) [20-23], and those on the implant tip and femoral cortical impact, mainly focus on the intramedullary nail surgery. However, few studies have focused on the femoral prosthesis stem tip and femoral cortical impact problems after THA. $[24,25]$ In this study, the impact between the femoral stem tip and the femoral cortex and postoperative thigh pain were studied.

Our retrospective analysis of 172 patients undergoing primary THA showed that the cortical impaction of the distal femur was not uncommon after primary total hip arthroplasty, with an incidence of $14.5 \%$, especially in women of small stature. Vision (as women get older), osteoporosis, femoral cortical thinning, the femur bow angle would be increased, when the biological type femoral stem (tapered straight shank) was used. In order to ensure better proximal pressure distribution, the larger femoral prosthesis stem would be chosen, and the femoral stem length would be increased, thereby increasing the point between the cortex and the impact of risk within the plants.[26-28] In addition, the existing biological femoral stem prosthesis is mainly designed based on patients in European or American, and therefore the LK. LCU femoral stem prosthesis of the same length used in this study was bound to increase the risk of collision when implanted into the body of Asians. For Asian patients with short stature, and femoral bow angle in the proximal with suitable cases, shorter creature type femoral line handle THA may be beneficial. However, Gielis et al.[29-31] have found that using short shank primary 
total hip replacement, thigh pain would follow the surgery, which would not be significantly reduced. [2931] In addition, Boese et al. [32] have also reported that the mismatch between femoral morphology and femoral prosthesis geometry in total hip arthroplasty not only increases the surgical difficulty, but also bring serious implant-related complications. The selection of too large prosthesis during femoral stem implantation would lead to the difficulty of femoral stem implantation. Some physicians may reinsert the bone after further appropriate distention. However, excessive reaming could result in postoperative pain and other problems. The choice of a smaller femoral stem prosthesis may affect the stability of total hip arthroplasty. Moreover, it also increases the risks of complications such as postoperative pain and fractures around the prosthesis. $[6,33,34]$ Therefore, it is important to find out the risk factors associated with femoral stem and femoral cortex impact after primary THA. Preoperative planning can be made in advance, and attention should be paid to the choice of femoral stem prosthesis. Therefore, the personalized femoral stem placement schemes should be developed, to facilitate the placement, decrease the operation difficulty and reduce the occurrence of postoperative complications.

The femoral stem implant angle is another risk factor with great influence, which is easily overlooked. As Teloken et al. [35] have believed that the placement angle of the femoral stem prosthesis should be less than or equal to $3^{\circ}$ on anteroposterior hip X-ray. Otherwise, the prosthesis was considered to be in varus or valgus position. In this study, our results showed that the risk of collision between the femoral stalk tip and the cortex was significantly higher when the prosthesis was in varus, compared with when the prosthesis was not varus. Moreover, postoperative thigh pain was found to be mainly concentrated in the patients with inversion at 3-month follow-up. These patients had obvious postoperative thigh pain and lower postoperative Harris scores. Our results also showed that the contact area of such patients in Gruen zones 2 was relatively small. Similar findings have been obtained by Junya et al., who have shown that the contact area of Gruen 2 is negatively correlated with post-operative pain. [8] This may be largely related to the experience and technical level of the surgeon. Therefore, this phenomenon can be a good reminder to the surgeon. During THA, especially when the femoral stem is inserted, more attention should be paid to the implant angle. In order to avoid too large angle of implantation, which might result in the impact of the postoperative prosthesis and the distal femur cortex, and thus affect the surgical quality.

This study has some limitations. First, the relatively small sample size and the nature of retrospective study limited the level of evidence. Second, it was limited to LK-LCU type femoral stem prosthesis, which was difficult to explain the problems of other femoral stem prosthesis.

\section{Conclusions}

Although the THA technology is mature, with remarkable effects, the consequences caused by improper placement of the prosthesis should not be ignored. For example, the impact between the femoral stem prosthesis tip and the distal femoral cortex, a clinical phenomenon described herein should be paid more attention before surgery, especially in patients with short stature. It is suggested that the surgeon should strictly control the placement angle of the femoral stem prostheses, to reduce the occurrence of 
impingement, improve the repairing effect and increase the patient satisfaction, which would further promote the development of personalized treatments.

\section{Abbreviations}

THA: Total hip arthroplasty; HHS: Harris Hip score;

PIA: Prosthesis implant angle; BMI: Body mass index;

LK-LCU: LCU-PoroLink; VAS:Visual Analogue Scale;

CFI: canal-flare index.

\section{Declarations}

\section{Acknowledgements}

We would like to acknowledge the whole staff at Yijishan Hospital of Wannan Medical College for their help in accessing the data for this study.

\section{Authors'contributions}

Authors $\mathrm{CML}, \mathrm{CW}$ and $\mathrm{PBC}$ helped in collecting, tabulating and analysing the data from the hospital records. Author QW was the primary surgeon, who conceptualised the study and developed the protocols and was a major contributor for the study. Author YZW was primarily helpful in the statistical analysis. Authors $Y Z$ and DSW et al. helped in writing and reviewing the manuscript. All authors have contributed to the write up and read and approved the final manuscript.

\section{Funding}

This study did not receive any funding.

\section{Availability of data and materials}

The datasets used and/or analysed during the current study are available from the corresponding author on request.

\section{Consent for publication}

Agreement

\section{Competing interests}

The authors declare they have no competing interests. 


\section{Author details}

${ }^{1}$ Yijishan Hospital of Wannan Medical College ,Zheshan West Road, Wuhu, China

${ }^{2}$ Bone and Joint Surgery, Yijishan Hospital of Wannan Medical College ,Zheshan West Road, Wuhu, China

\section{Ethics approval and consent to participate}

The study was approved by the Ethics Committee of the First Affiliated Hospital of Wannan Medical College.

\section{References}

1. Tilbury C, et al. Unfulfilled Expectations After Total Hip and Knee Arthroplasty Surgery: There Is a Need for Better Preoperative Patient Information and Education. J Arthroplasty. 2016;31(10):213945 .

2. Klit J. Results of total joint arthroplasty and joint preserving surgery in younger patients evaluated by alternative outcome measures. Dan Med J. 2014;61(4):B4836.

3. Adelani MA, et al. Has total hip arthroplasty in patients 30 years or younger improved? A systematic review. Clin Orthop Relat Res. 2013;471(8):2595-601.

4. Ezquerra L, et al. Range of Movement for Impingement and Dislocation Avoidance in Total Hip Replacement Predicted by Finite Element Model. J Med Biol Eng. 2017;37(1):26-34.

5. Gala L, Clohisy JC, Beaulé PE. Hip Dysplasia in the Young Adult. J Bone Joint Surg Am. 2016;98(1):63-73.

6. Brown TE, et al. Thigh pain after cementless total hip arthroplasty: evaluation and management. J Am Acad Orthop Surg. 2002;10(6):385-92.

7. Baert IAC, et al. Short stem total hip arthroplasty: Potential explanations for persistent post-surgical thigh pain. Med Hypotheses. 2017;107:45-50.

8. Yoshitani $\mathrm{J}$, et al. The use of density mapping in the analysis of thigh pain after total hip arthroplasty in patients with well-fixed tapered wedge stems. J Orthop Surg (Hong Kong). 2020;28(2):2309499020930306.

9. Elkins JM, Callaghan JJ, Brown TD. The 2014 Frank Stinchfield Award: The 'landing zone' for wear and stability in total hip arthroplasty is smaller than we thought: a computational analysis. Clin Orthop Relat Res. 2015;473(2):441-52.

10. Jo WL, et al. Frequency, Developing Time, Intensity, Duration, and Functional Score of Thigh Pain After Cementless Total Hip Arthroplasty. J Arthroplasty. 2016;31(6):1279-82.

11. Abdel MP, et al. What Safe Zone? The Vast Majority of Dislocated THAs Are Within the Lewinnek Safe Zone for Acetabular Component Position. Clin Orthop Relat Res. 2016;474(2):386-91. 
12. Tezuka T, et al. Functional Safe Zone Is Superior to the Lewinnek Safe Zone for Total Hip Arthroplasty: Why the Lewinnek Safe Zone Is Not Always Predictive of Stability. J Arthroplasty. 2019;34(1):3-8.

13. Young EY, et al. Are the left and right proximal femurs symmetric? Clin Orthop Relat Res. 2013;471(5):1593-601.

14. Barrack RL, et al. Patients' perception of pain after total hip arthroplasty. J Arthroplasty. 2000;15(5):590-6.

15. Dorr LD, et al. Structural and cellular assessment of bone quality of proximal femur. Bone. 1993;14(3):231-42.

16. Noble P, et al., The anatomic basis of femoral component design. 1988(235): p. 148-65.

17. Lavernia $C$, et al. Thigh pain in primary total hip arthroplasty: the effects of elastic moduli. J Arthroplasty. 2004;19(7 Suppl 2):10-6.

18. Vresilovic EJ, Hozack WJ, Rothman RH. Incidence of thigh pain after uncemented total hip arthroplasty as a function of femoral stem size. J Arthroplasty. 1996;11(3):304-11.

19. Burkart BC, et al. Thigh pain in cementless total hip arthroplasty. A comparison of two systems at 2 years' follow-up. Orthop Clin North Am. 1993;24(4):645-53.

20. Trisolino G, et al. Labral calcification plays a key role in hip pain and symptoms in femoroacetabular impingement. J Orthop Surg Res. 2020;15(1):86.

21. Yoshitani J, et al. Anatomic stem inserted according to native anteversion could reproduce the native anterior distance of the femoral head and decrease bony impingement in total hip arthroplasty. Int Orthop. 2020;44(2):245-51.

22. Qiu J, et al. Risk factors for iliopsoas impingement after total hip arthroplasty using a collared femoral prosthesis. J Orthop Surg Res. 2020;15(1):267.

23. Yao JJ, et al. What Is the Survivorship After Hip Arthroscopy for Femoroacetabular Impingement? A Large-database Study. Clin Orthop Relat Res; 2020.

24. Roberts JW, Libet LA, Wolinsky PR. Who is in danger? Impingement and penetration of the anterior cortex of the distal femur during intramedullary nailing of proximal femur fractures: preoperatively measurable risk factors. J Trauma Acute Care Surg. 2012;73(1):249-54.

25. Shetty A, Shenoy PM, Swaminathan R. Mismatch of long Gamma intramedullary nail with bow of the femur: Does radius of curvature of the nail increase risk of distal femoral complications? J Clin Orthop Trauma. 2019;10(2):302-4.

26. Su X, et al., Three-Dimensional Analysis of the Characteristics of the Femoral Canal Isthmus: An Anatomical Study. 2015. 2015: p. 459612.

27. Thiesen DM, et al. Femoral antecurvation-A 3D CT Analysis of 1232 adult femurs. PLoS One. 2018;13(10):e0204961.

28. Zhang JZ, et al. Age-related dynamic deformation of the femoral shaft and associated osteoporotic factors: a retrospective study in Chinese adults. Arch Osteoporos. 2020;15(1):157. 
29. Suksathien Y, Sueajui J. Mid-term results of short stem total hip arthroplasty in patients with osteonecrosis of the femoral head. Hip Int. 2019;29(6):603-8.

30. Amendola RL, et al. Two- to 4-Year Followup of a Short Stem THA Construct: Excellent Fixation, Thigh Pain a Concern. Clin Orthop Relat Res. 2017;475(2):375-83.

31. Gielis W, et al. Increased Persistent Mid-Thigh Pain After Short-Stem Compared With Wedge-Shaped Straight-Stem Uncemented Total Hip Arthroplasty at Medium-Term Follow-Up: A Randomized DoubleBlinded Cross-Sectional Study. The journal of arthroplasty. 2019;34(5):912-9.

32. Boese CK, et al. Agreement Between Proximal Femoral Geometry and Component Design in Total Hip Arthroplasty: Implications for Implant Choice. J Arthroplasty. 2016;31(8):1842-8.

33. Pierannunzii LM, Thigh pain after total hip replacement: a pathophysiological review and a comprehensive classification. Orthopedics, 2008. 31(7): p. 691-9; quiz 700-1.

34. Oh KJ, et al. Total hip arthroplasty using the miniature Anatomic Medullary Locking stem. Clin Orthop Relat Res. 2006;447:85-91.

35. Teloken MA, et al. Ten to fifteen-year follow-up after total hip arthroplasty with a tapered cobaltchromium femoral component (tri-lock) inserted without cement. J Bone Joint Surg Am. 2002;84(12):2140-4.

\section{Figures}
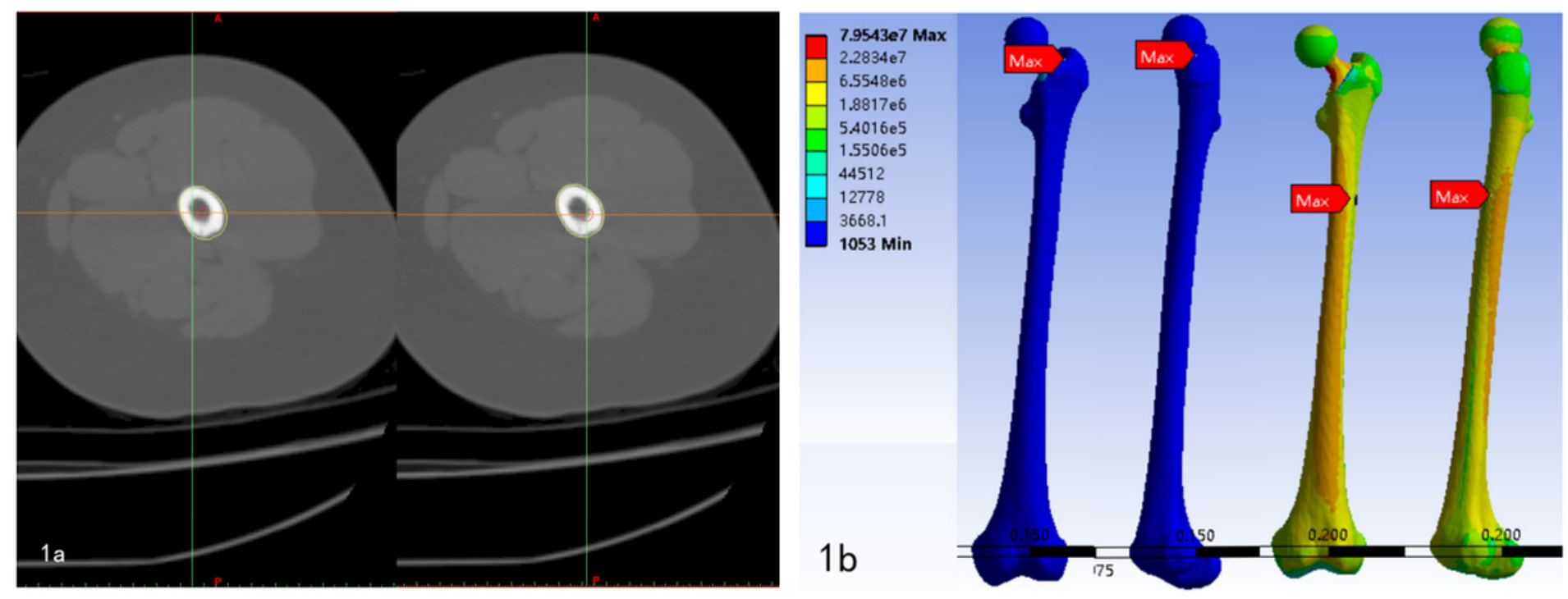

Figure 1

(1a) Axial CT view of the femoral stem prosthesis tip with a hollow on the left and contact with the cortex on the right. (1b) The overall force analyses of the hollow femoral stem group in the positive and lateral position (left), and the femoral stem tip contact group in the positive and lateral position (right). 


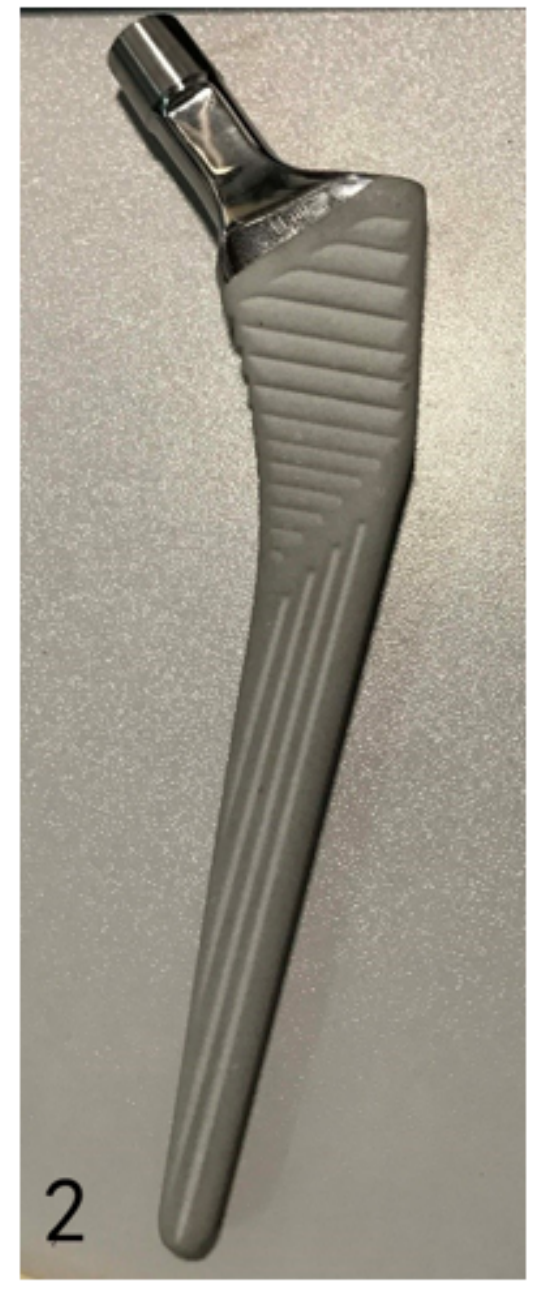

Figure 2

LINK.LCU stem was a tapered wedge stem with a fully coated surface treatment. 


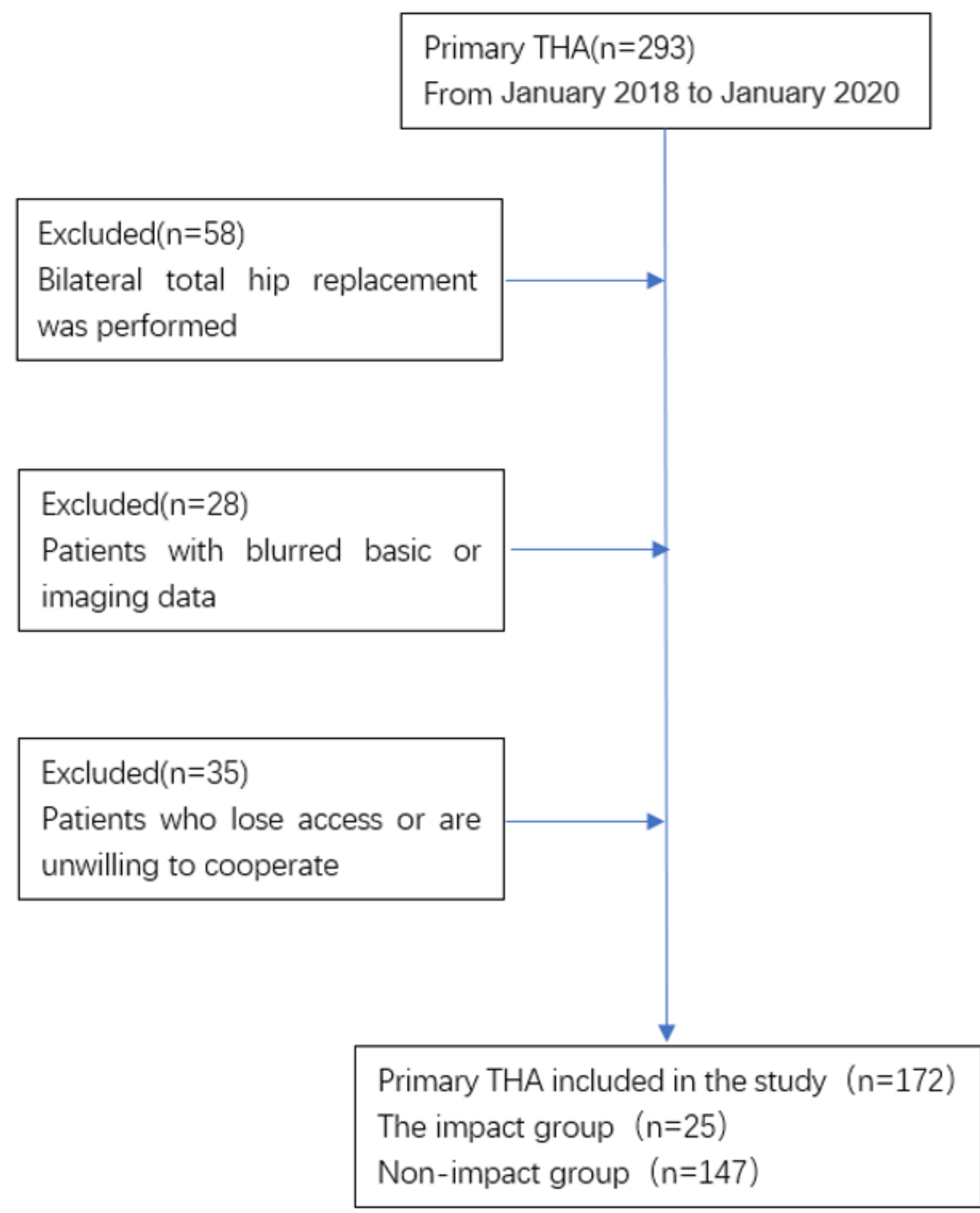

Figure 3

Patient selection flow chart. 


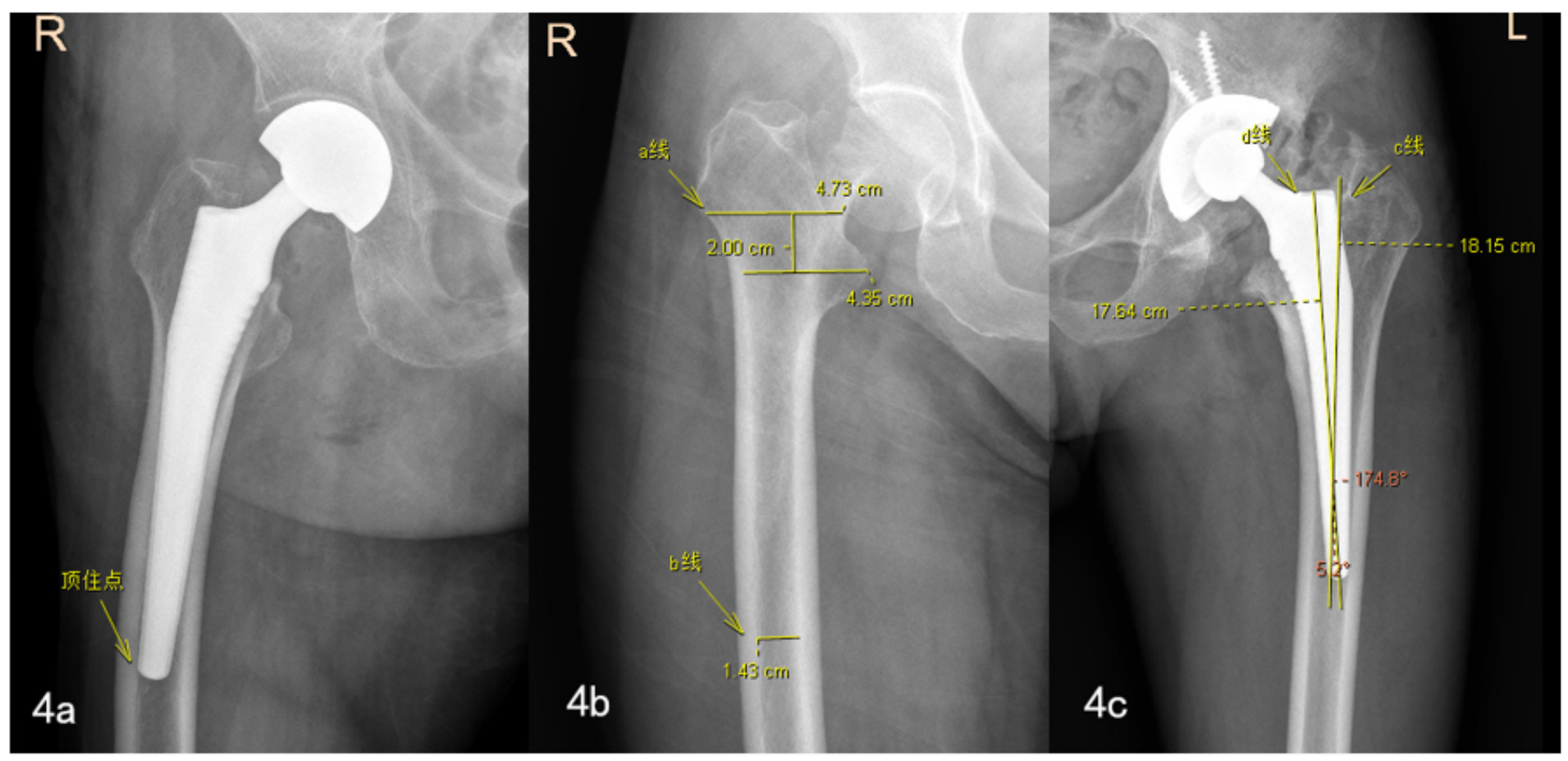

\section{Figure 4}

a. Anterior X-ray images of the hip joint after surgery. The large lateral arch of the femur caused the femoral stem tip to collide with the lateral cortex of the femur. The point of impact was indicated by the arrow. b. Anterior X-ray images of the femur before surgery. Line a was the width of the medullary cavity at 2-cm proximal to the lesser trochanter. Line $b$ was the width of the medullary cavity of the femoral isthmus. $\mathrm{CFI}=\mathrm{a} / \mathrm{b}$. CFI, canal-flare index. c. DR radiographs of the orthotopic hip joint after surgery. Line $\mathrm{C}$ was the long axis of femoral shaft. Line $d$ was the long axis passing through the upper vertex of the femoral stem and the midpoint of the lower tip. The angle between lines $c$ and $d$ was the prosthesis implant angle (PIA) . 


\section{The pain of patients}

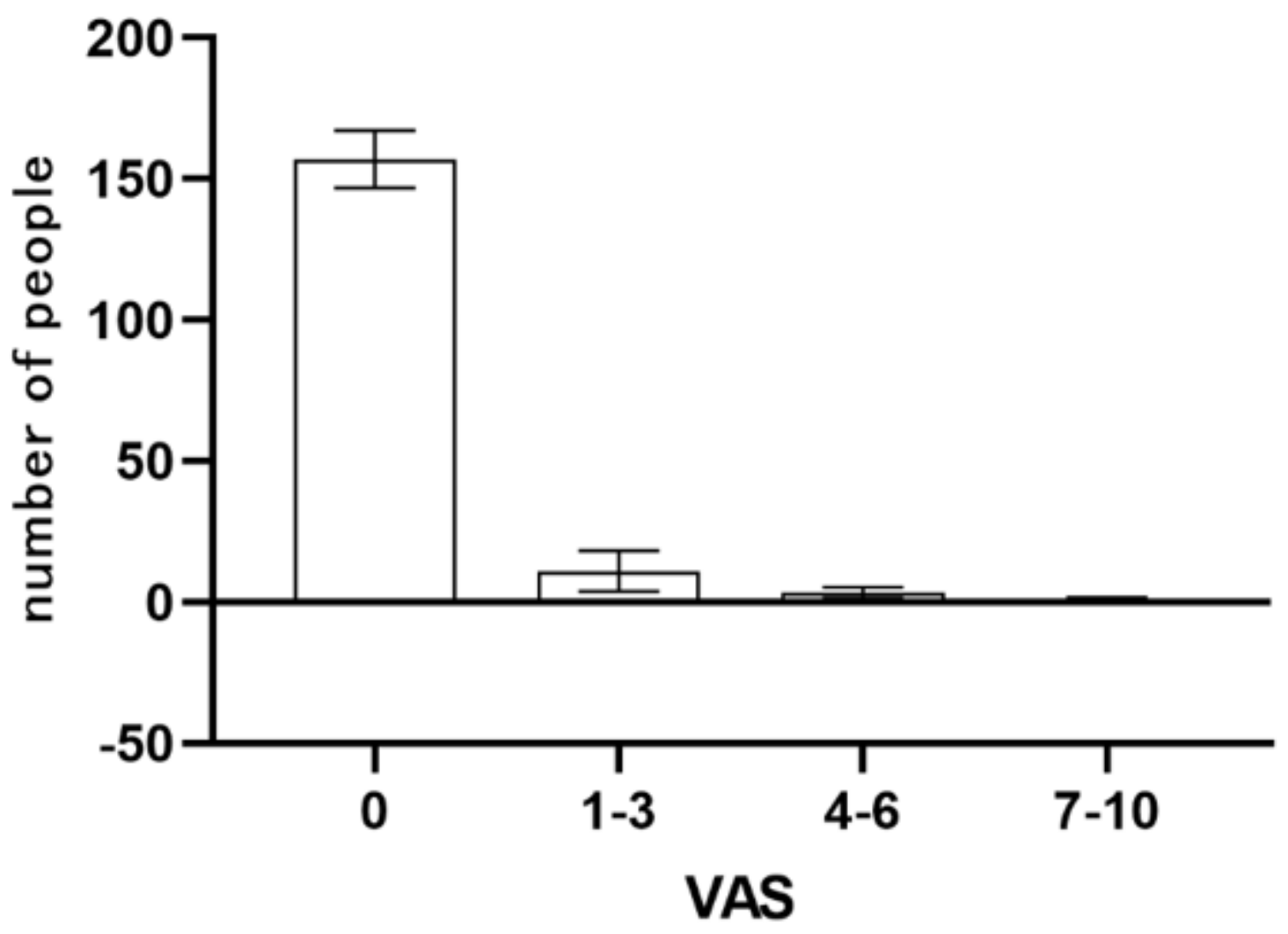

Figure 5

Number distribution of VAS. 